\title{
First Double Star Observations in the HIPPARCOS Tycho Project
}

\author{
JEAN-LOUIS HALBWACHS \\ CDS, URA 1280, Observatoire de Strasbourg 11, rue de l'Universite, \\ F-67000 Strasbourg, France
}

ABSTRACT: The Tycho project will provide positions and $(B, V)$ magnitudes for about 1 million stars, and will be complete for stars brighter than magnitude $(B+V) / 2$ $=10.5$. The components of double stars will obtain individual measurements when the separations are wider than $3^{\prime \prime}$.

\section{INTRODUCTION}

The aim of Tycho data analysis is to derive the positions and the photometric data from the observations by the star mapper of the HIPPARCOS satellite. The positions of 1 million stars will be obtained with an accuracy of 0 ".03. Half a million stars will have $B$ and $V$ magnitudes (with an accuracy of 0.03 magnitude when $B=10.5$ ), and the other 500,000 stars will receive a combined $(B+V)$ magnitude, hereafter called $\mathrm{T}$. The organization of the project and an assessment based on the first observations of the satellite were presented in $\mathrm{H} \varnothing \mathrm{g}$ et al. (1992).

The star mapper of the HIPPARCOS satellite is equiped with two groups of slits with different slopes: The "vertical" group is perpendicular to the direction of scanning of the satellite, and the "chevron" group has two branches with inclinations of plus or minus 45 degrees to the scanning direction. The transits of stars accross the slits are recorded with two photomultiplier tubes, one for $B$ and one for the $V$ colour band.

The stars of the program are selected in two steps. A "Tycho Input Catalogue", called "TIC" hereafter, was prepared (Egret et al. 1992). The INCA database of the HIPPARCOS program provided the bright stars, and the faint stars were added from the Guide Star Catalogue of the Hubble Space Telescope. The TIC contains 3 million stars, although only 1 million stars should be bright enough to be kept in the final catalogue; but the magnitudes of the stars are not accurate, and it was necessary to use a faint limit in magnitude in order to be sure that nearly all the stars that may be observed by the instrument were included in the TIC.

The second step in selecting the stars is a process called "Recognition" (Halbwachs et al. 1992), that will be completed by the end of this year (1992). The recognition is based on the observations of the stars in the TIC that were obtained during the first year of the scientific mission. Its first purpose is to select the TIC-stars that were detected, and to derive their positions with an accuracy better than $0^{\prime \prime} .2$; secondly, recognition will add new stars or components omitted in the TIC, when they are less than $20^{\prime \prime}$ from a TIC position. The benefit of the Tycho project on our knowledge of double stars depends therefore essentially on the efficiency of recognition. This question will be investigated hereafter, on the basis of first observations done by the satellite. 


\section{THE RECOGNITION PROCESS}

This processing task was fully presented in Halbwachs et al. (1992). Since it is too complicated to be completely explained again, only some basic principles are summarized here.

Recognition is an automatic mapping process. $20^{\prime \prime}$-radius maps are generated around the positions of the 3 millions star in the TIC. Each map is analyzed numerically, without use of any graphical means. The detections found in the photon counts of the star mapper are represented on the maps by lines, called "detection lines" hereafter. The detection lines indicate the positions of the slit groups of the star mapper at the epochs of the detections; Each line receives a weight, that is related to the signal-to-noise ratio of the detection. The positions of the stars are indicated in a map by the intersections of detection lines.

In order to facilitate the recognition of the TIC stars, the detection threshold, i.e. the minimum signal-to-noise ratio, is a little smaller inside a $6^{\prime \prime}$-radius area around the center of the map than in the outer part. Moreover, the stars in the inner part are searched before those in the outer part.

This process is illustrated in Figure 1. The TIC star 4956:342 was recognized near the center of the map, and two other stars were recognized in the outer part of the map. At least one of these two stars is true, since it is near a position in the TIC. The other star may be a companion missing in the TIC, or a false star generated by background lines. If it is a false star, it will be discarded when more observations will become available.

\section{A MINIMUM ESTIMATION OF THE EFFICIENCY OF DOUBLE STAR RECOGNITION}

The proportion of stars having a companion in the TIC closer than $20^{\prime \prime}$ is $2.5 \%$. In order to evaluate the completeness of Tycho for double stars, two questions are to be considered:

- What is the proportion of companions in the TIC that are really recognized?

- What is the proportion of secondary components that could be added by recognition if they were omitted in the TIC?

Since the recognition process is not completed yet, only a small sample and a small number of observations are available for an assessment. This sample contains 99 double stars having accurate positions in the TIC, and with components brighter than $T=10.5 \mathrm{mag}$. The separations are between $3^{\prime \prime}$ and $20^{\prime \prime}$, since the detection process cannot separate stars closer than 2 arcsec, when the direction of scanning is parallel to the position angle. Each star has at least 16 observations, with a median number of 20 . This is only half the average number of observations that will be used in the nominal recognition. The number of scanning directions is also smaller than nominal, since the greatest part of these stars were observed with the same scanning direction. This is a serious limitation, since one scanning direction may generate lines with at most three different slopes. 


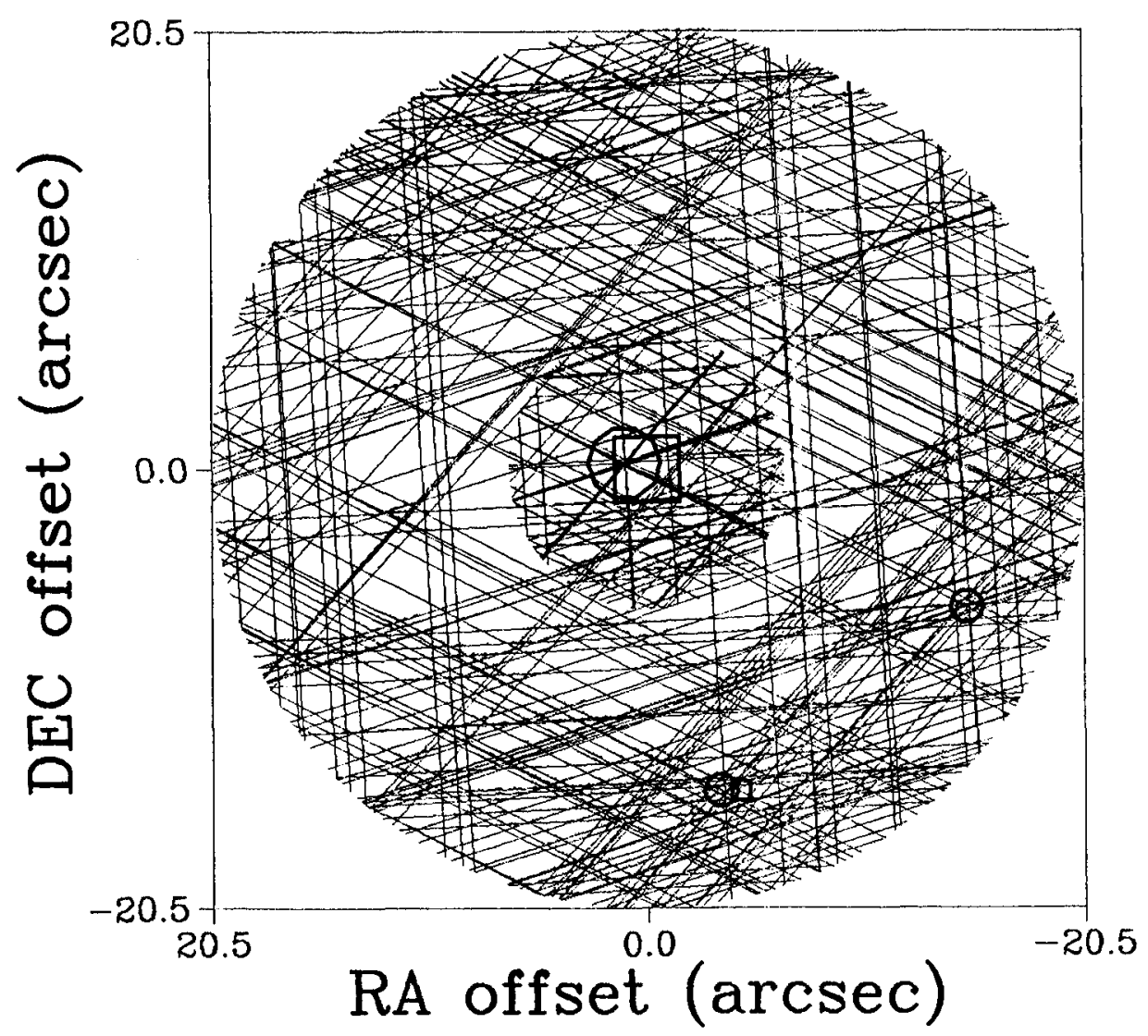

FIGURE 1. The recognition map of TIC 4956:342. 21 observations with 2 directions of scanning are represented in this map. The recognized stars are represented by circles, and the squares indicate the positions in the TIC. The magnitude of the central star is $\mathrm{T}=9.05$. The secondary component below is TIC 4956:10003; its magnitude is $T=11.75$. The separation is $15^{\prime \prime} .6$. 
The proportions of stars that were recognized in their own recognition map are given in Table 1 . It appears that $81 \%$ of the components were recognized, and that both components were found for two-thirds of the double stars.

TABLE 1. Recognition of double star components of the TIC, with a median number of only 20 observations per star and at most two scanning directions.

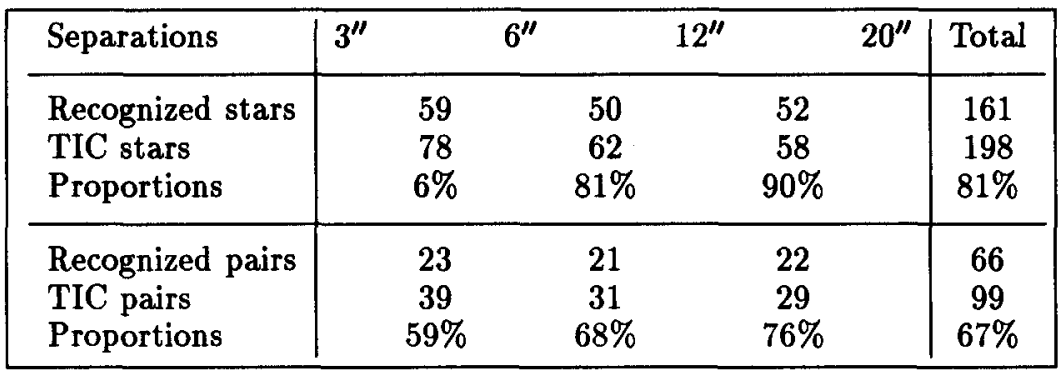

The ability to recognize a double star in the recognition map of the primary component is estimated in Table 2 . The proportion of primary components that were found in the right places was $88 \%$, but only $42 \%$ of the secondaries were correctly recognized. The proportion of recognized double stars is then $39 \%$. Contrary to Table 1, the proportion is larger in the closest bin in separation than in the other ones. This is due to the small detection threshold that was used in the inner part of the map.

TABLE 2. Recognition in the map of the primary component, with a median number of only 20 observations per star and at most two scanning directions.

\begin{tabular}{|c|c|c|c|c|}
\hline Separations & $3^{\prime \prime}$ & $6^{\prime \prime}$ & $20^{\prime \prime}$ & Total \\
\hline $\begin{array}{l}\text { Recogn. primaries } \\
\text { TIC primaries } \\
\text { Proportions }\end{array}$ & $\begin{array}{c}33 \\
39 \\
85 \%\end{array}$ & $\begin{array}{c}28 \\
31 \\
90 \%\end{array}$ & $\begin{array}{c}27 \\
29 \\
93 \%\end{array}$ & $\begin{array}{c}88 \\
99 \\
88 \%\end{array}$ \\
\hline $\begin{array}{l}\text { Recogn. secondaries } \\
\text { TIC secondaries } \\
\text { Proportions }\end{array}$ & $\begin{array}{c}18 \\
39 \\
46 \%\end{array}$ & $\begin{array}{c}11 \\
31 \\
35 \%\end{array}$ & $\begin{array}{c}13 \\
29 \\
44 \%\end{array}$ & $\begin{array}{c}42 \\
99 \\
42 \%\end{array}$ \\
\hline $\begin{array}{l}\text { Recogn. pairs } \\
\text { TIC pairs } \\
\text { Proportions }\end{array}$ & $\begin{array}{c}17 \\
39 \\
44 \%\end{array}$ & $\begin{array}{c}10 \\
31 \\
32 \%\end{array}$ & $\begin{array}{c}12 \\
29 \\
41 \%\end{array}$ & $\begin{array}{c}39 \\
99 \\
39 \%\end{array}$ \\
\hline
\end{tabular}




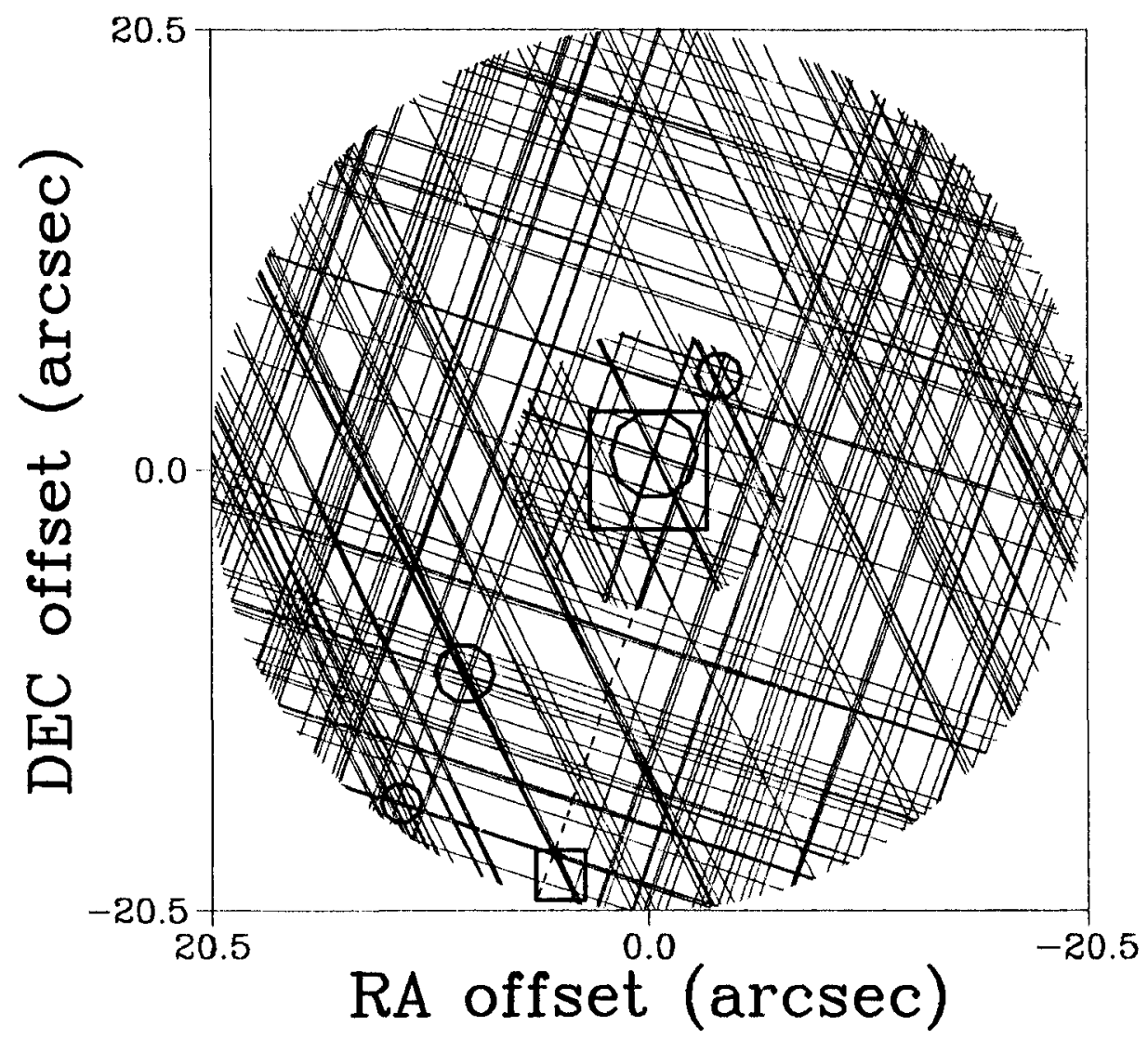

FIGURE 2. The recognition map of TIC 4376:10003. 21 observations with the same scanning direction are represented. The T-magnitude of the central star is 7.8 . The secondary component was lost, although its place is visible in the bottom of the map, near the cross indicating its TIC position. This star is TIC 4376:10004, with magnitude $T=9.70$. The separation between the components is $19^{\prime \prime} .3$ 
These results are provisional and pessimistic since they are only an estimation of the rate of double stars that will be recognized on the basis of 20 observations and one direction of scanning. In the nominal recognition, only a few percent of stars will be so poorly observed. Although it is still not possible to estimate the recognition rate for the other stars, it is easy to explain why a unique scanning direction implies that so many double stars where lost. A typical example is given in Figure 2: the TIC star 4376:10003 was recognized in the center of the map, but another star was also found in the inner map. This star was obviously false, since it was generated by the intersection of background lines and some detection lines of the true secondary component that was in the outer part of the map. The lines related to the recognized stars were no more taken into account when the outer part was treated, and the secondary component was not recognized since it had lost one of its three groups of lines. But the two remaining groups of detection lines generated two other false stars - The situation will improve much when more detection lines with different slopes will be considered: the false star in the inner map will not be selected, since it will become fainter than the recognition threshold, and, even if some detection lines having the same slope would be discarded, the remaining ones would be enough for finding the true star. This assumption should be verified before the end of this year, when the recognition process will be completed.

\section{ACKNOWLEDGMENTS}

It is a pleasure to thank Prof. E. Høg for correcting the manuscript.

\section{REFERENCES}

Egret D., Didelon P., McLean B.J., Russel J.A., \& Turon C. 1992, A\&A, 258 Halbwachs J.L., Hog E., Bastian U., Hansen P.C., \& Schwekendiek P. 1992, $A \& A, 258$ Høg E., Bastian U., Egret D., et al. 1992, A\&A, 258 